67th International Astronautical Congress (IAC), Guadalajara, Mexico, 26-30 September 2016.

Copyright (C) 2016 by the International Astronautical Federation (IAF). All rights reserved.

IAC-16-C2.6.6.32493

\title{
TEMPERATURE RESTRICTIONS FOR MATERIALS USED IN AEROSPACE INDUSTRY FOR THE NEAR-SUN ORBITS
}

\author{
Elena Ancona ${ }^{\mathrm{a}}$ and Roman Ya. Kezerashvili ${ }^{\mathrm{b}}$ \\ ${ }^{\text {aP }}$ olitecnico di Torino, Corso Duca degli Abruzzi 24, TO, 10129, Torino, Italy, elena.ancona@gmail.com \\ ${ }^{\mathrm{b}}$ Department of Physics, New York City College of Technology, The City University of New York, 300 Jay \\ Street, Brooklyn NY, 11201, New York City, USA, rkezerashvili@citytech.cuny.edu
}

\begin{abstract}
For near-Sun missions, the spacecraft approaches very close to the Sun and space environmental effects become relevant. Strong restrictions on how much close it can get derive from the maximum temperature that the used materials can stand, in order not to compromise the spacecraft's activity and functionalities. In other words, the minimum perihelion distance of a given mission can be determined based on the materials' temperature restrictions. The temperature of an object in space depends on its optical properties: reflectivity, absorptivity, transmissivity, and emissivity. Usually, it is considered as an approximation that the optical properties of materials are constant. However, emissivity depends on temperature. The consideration of the temperature dependence of emissivity and conductivity of materials used in the aerospace industry leads to the conclusion that the temperature dependence on the heliocentric distance is different from the case of constant optical properties [1. Particularly, taking into account that emissivity is directly proportional to the temperature, the temperature of an object increases as $r^{-2 / 5}$ when the heliocentric distance $r$ decreases. This means that the same temperature will actually be reached at a different distance and, eventually, the spacecraft will be allowed to approach closer to the Sun without compromising its activities. We focused on metals used for aerospace structures $(\mathrm{Al}, \mathrm{Ti})$, however our analysis can be extended to all kinds of composite materials, once their optical properties - in particular emissivity - are defined.
\end{abstract}

Keywords: Near-Sun orbits, temperature restrictions for materials.

\section{Introduction}

Materials in space are nowadays a key topic of research for every aerospace industry. In fact, their characteristics and performances are extremely relevant for every feature of the mission, including the cost: the main objective is to obtain very light materials with great mechanical properties. Aluminum has been for long time a valid compromise for its weight and good characteristics, however the actual trend is to shift to carbon fiber and composite structures, and possibly in the future to combinations of plastics and various hybrid materials such as metal-matrix composites, that will greatly reduce the weight of a spacecraft and also its launch costs. Indeed, carbon fiber has already replaced many spacecraft components, except for bulkheads that are still made from titanium, aluminum or other conventional metals and alloys because of the tremendous thermal and mechanical demands. All materials have a well defined range of temperature in which their behavior is optimal. Out of these bounds, their properties' degradation leads to failing performances. Temperature is one of the most considerable drivers, especially for Near-Sun missions, with electromagnetic radiation and solar wind. When approaching the Sun, the increasing temperature could become unbearable, depending on the specific material characteristics. Moreover, the temperature reached by the spacecraft depends not only on the outer environment, but also on the spacecraft itself. In particular, the spacecraft tem- 
67th International Astronautical Congress (IAC), Guadalajara, Mexico, 26-30 September 2016

Copyright (C) 2016 by the International Astronautical Federation (IAF). All rights reserved.

perature is function of its materials' optical properties, that in turn are function of temperature. It is clear, then, that a better knowledge of the materials' optical properties dependence on temperature could be the key to design properly a Near-Sun mission. Of course, consideration of optical degradation of materials due to proton and electron radiation for a Near-Sun mission is very important, however we do not address this issue in the present study.

This paper is organized in the following way: in Section 2 a brief overview of the most commonly used materials in space applications is given, while Section 3 provides the key features of temperature dependence of a spacecraft material on heliocentric distance. Our analysis and results for metals are reported in Section 4 , whereas Section 5 suggests plausible future developments of the study. Conclusions follow in Section 6 .

\section{Materials in space}

The principal design driver for a spacecraft is weight: the main challenge is to minimize mass, and consequently launch costs, without compromising reliability and functionality. Moreover, a spacecraft must accommodate the payload and its subsystems, satisfying the mounting requirements, and then support itself and its payload through all phases of the mission, included the launch. In particular, not only good stiffness and strength properties are required, but also oscillation and resonance frequencies of structures must be taken into account. Hence, the design of spacecraft structures needs an extremely careful selection of materials based upon their strength, stiffness, damage tolerance, thermal and electrical properties, as well as corrosion resistance and shielding capabilities. For this reason, the space sector has traditionally been a promoter for the development and the application of advanced engineering materials. Our study has been conducted for the two most vastly used metals in aerospace industry: in fact, considering their strength and density, it is clear why aluminum and titanium are the preferred for lightweight aerospace alloys. Titanium alloys are used where lighter aluminum alloys no longer meet strength, corrosion resistance and elevated temperature requirements [2], as aluminum has a fusion temperature of $660{ }^{\circ} \mathrm{C}$, whereas titanium of $1668{ }^{\circ} \mathrm{C}$. Aluminum has a low density, good specific strength and it is easily workable. For its range of applicability it is also cheap and widely available. However, its weak spot is the low melting point. Titanium is more expansive but guar- antees better performance in hostile environments due to its great corrosion resistance. Moreover, it can bear high temperatures.

\section{Temperature dependence on heliocentric distance}

It might be convenient to remark the difference between environmental temperature in space and the temperature of an object, such as a spacecraft, in space.

\subsection{Environmental temperature in space}

Matter in space is extremely concentrated into celestial bodies. The space between them could be considered as a near-vacuum, where particles may be many miles apart. Hence, under outer space conditions, almost no energy is transferred "directly" because of the vast distances involved. As a result, radiation is effectively the only heat-exchanging mechanism in most of the space environment. In fact, the average temperature of interplanetary space is $2.7 \mathrm{~K}$, due to the cosmic microwave background.

\subsection{Spacecraft temperature dependence on heliocentric distance}

An object in space radiates heat and receives heat radiated from other bodies. The result of this energy balance is that if the considered body radiates more heat than it receives it will cool down; on the contrary, if the incoming heat is more than that radiated, it will warm up. As the intensity of received radiation decreases with the square of the distance from the energy source, a spacecraft approaching the Sun will be exposed to higher level of electromagnetic radiation. Distance from stars and radiation exposure are the prime temperature determinants for an object in space. In fact, if one side of a spacecraft is exposed to direct sunlight and radiation, while the other side is shadowed and facing out into deep space, the spacecraft would suffer an extreme temperature differential which, if not sapiently contrasted, could be dangerous and critical for the system survival.

It is clear then that the temperature of a spacecraft depends on its optical properties: reflectivity, absorptivity, transmissivity and emissivity. In fact, the solar electromagnetic radiation can be reflected, absorbed or transmitted. Therefore, one can write:

$$
\rho(\lambda, T)+\alpha(\lambda, T)+\tau(\lambda, T)=1,
$$


where $\rho(\lambda, T), \alpha(\lambda, T)$ and $\tau(\lambda, T)$ are the radiative or optical properties of the material: spectral (as they depend on wavelength $\lambda$ ) hemispherical (as they are not directional) reflectivity, absorptivity and transmissivity, respectively [3]. The contribution of transmissivity $\tau$ can be neglected, as experimental data confirm it is a very small fraction (almost $2 \%$ ) of the incoming solar energy flux. Indeed, once that energy has been absorbed, it can also be emitted from the surface, as a secondary process. From the Stefan-Boltzmann's law, the rate of energy emitted from a surface at a certain temperature is proportional to the fourth power of the temperature.

In order to estimate the temperature of a body's surface its effective temperature is commonly used. The effective temperature of a generic object is defined as the temperature of a black body that would emit the same total amount of electromagnetic radiation [4]:

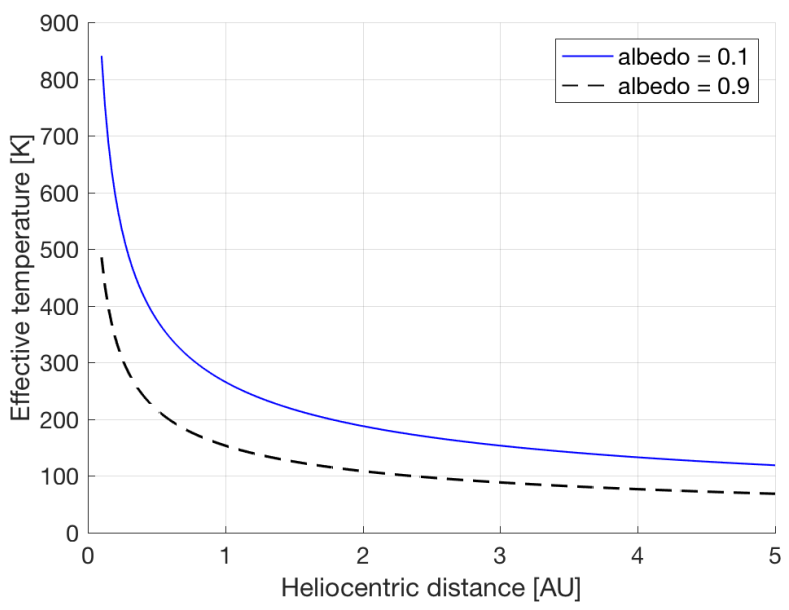

Figure 1: Temperature dependence on reflectivity. The graph shows the effective temperature of a body with different values of reflectivity (albedo). It is the temperature of a black body $(\varepsilon=1)$ that would emit the same total amount of electromagnetic radiation. If the body has lower emissivity, its actual temperature will be higher of $T_{\text {eff }}$. The solid curve represents a body with low reflectivity $a=0.1$, whereas the dashed curve a body with high reflectivity $a=0.9$. The effective temperature of a body with generic albedo would be between these limits.

$$
T_{e f f}=\left(\frac{L(1-a)}{16 \pi \sigma r^{2}}\right)^{1 / 4},
$$

where $L$ is the star's luminosity, $a$ is the albedo, $\sigma=$ $5.67 \cdot 10^{-8} \mathrm{Wm}^{-2} \mathrm{~K}^{-4}$ is the Stefan-Boltzmann constant and $r$ is the distance of the object from the star, all in SI units. The luminosity of a star depends on its radius $R_{S}$ and surface temperature $T_{S}$ :

$$
L=4 \pi \sigma R_{S}^{2} T_{S}^{4} .
$$

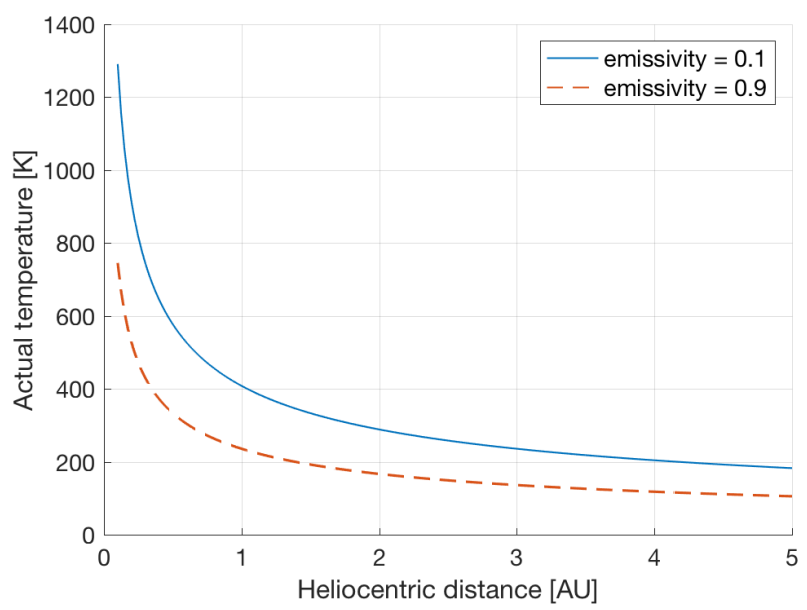

Figure 2: Temperature dependence on emissivity. The graph shows the actual temperature of a generic body with defined albedo $a=0.5$, for different values of emissivity. The solid curve represents a body with low emissivity $\varepsilon=0.1$, whereas the dashed curve a body with high emissivity $\varepsilon=0.9$. The actual temperature of a body with generic emissivity $0.1<\varepsilon<0.9$ would be between these limits.

For what concerns the albedo, it is a reflection coefficient, defined as the ratio of radiation reflected from the surface to the incident radiation, so $a=0$ for a perfectly black surface and $a=1$ for a perfectly white surface ${ }^{1}$ Fig. 1 shows the effective temperature dependence on heliocentric distance for different values of albedo. Eq. (2) is useful when the real emissivity of the specific body is unknown. However, it can be modified to account for the actual emissivity of a body $\varepsilon$, leading to more accurate results:

\footnotetext{
${ }^{1}$ The albedo changes in the range $0-1$ depending on the surface material, e.g. $a=0.06$ for the Moon, which main surface component is basalt, $a=0.16$ for Mars (iron oxide), $a=0.38$ for Earth, and $a=0.70$ for Jupiter (gas).
} 


$$
T_{a c t}=\left(\frac{A_{a b s}}{A_{\text {rad }}} \frac{L(1-a)}{4 \pi \sigma \varepsilon r^{2}}\right)^{1 / 4},
$$

where $A_{a b s}$ and $A_{\text {rad }}$ are the portion of the total area involved into absorption and radiation, respectively.

Eq. (4) allows to evaluate the surface temperature of celestial bodies. The ratio $A_{a b s} / A_{\text {rad }}$ is assumed to be $1 / 4$ for a rapidly rotating body and $1 / 2$ for a slowly rotating body. Note that the net emissivity of the planet may be lower due to surface or atmospheric properties, such as the greenhouse effect. When the planet's net emissivity in the relevant wavelength band is less than unity (less than that of a black body), the actual temperature of the body will be higher than the effective temperature: $T_{a c t}>T_{\text {eff }}$. In other words, $T_{\text {eff }}$ is the lower limit for the temperature of a body with a given reflectivity ratio, at a distance $r$ from a star of luminosity $L$. The actual temperature of a body with a mean value of reflectivity coefficient $(a=0.5)$ is shown in Fig. 2, for two different values of emissivity.

Eq. (4) is sometimes written in a more functional form 2 .

$$
T_{a c t}=273\left(\frac{\mathfrak{L}(1-a)}{\varepsilon \mathrm{r}^{2}}\right)^{1 / 4},
$$

where $\mathfrak{L}=L / L_{S U N}$ is the luminosity of a generic star in multiples of the Sun's power and $r$ is the distance between the body and the star in AU. As a result, the temperature of an object increases as $r^{-1 / 2}$ when the heliocentric distance decreases, if all the other parameters are constant.

\section{Temperature dependence on heliocentric distance for metals}

Let us focus our attention on metals, largely used in the aerospace industry.

In Section 3 a method for evaluating the surface temperature of a body has been provided: Eq. (4) can be applied to any material, once its constant emissivity and reflectivity are known. It requires as input the emissivity and reflectivity of the material, and gives as result its surface temperature. However recent studies show that reflectivity and emissivity also depend on temperature [5].

\footnotetext{
${ }^{2}$ http://spacemath.gsfc.nasa.gov.
}

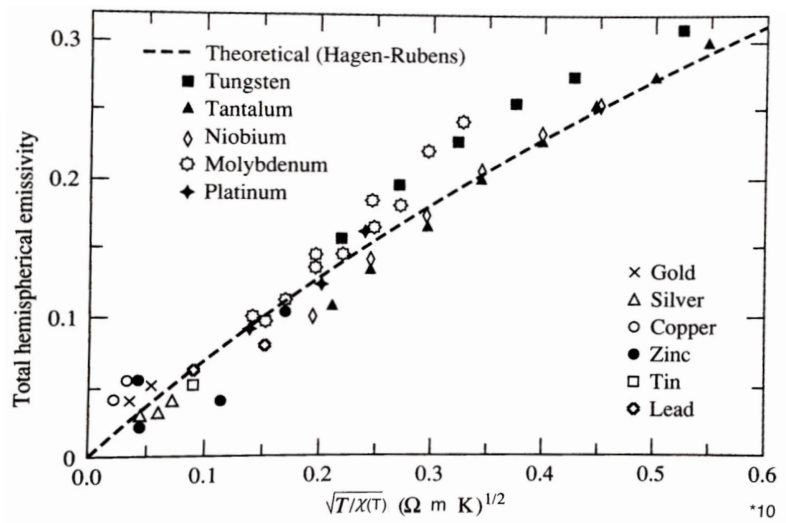

Figure 3: Total hemispherical emissivity of various metals as function of $\sqrt{T / \chi(T)}$, where $\chi$ is the material's conductivity [6].

\subsection{Optical parameters dependence on temperature}

In Eqs. (2), (4) and (5) the optical coefficients have been considered constant and not variable with time or temperature itself. Although reflectivity dependence on temperature can be neglected, the same doesn't apply for emissivity, which is directly proportional to the temperature, as suggested by Parker and Abbott [7, because electrical conductivity is inversely proportional to $T$. The expression they found for the total hemispherical emissivity for metals is the following:

$$
\begin{aligned}
\varepsilon(T)= & 7.66 \sqrt{\frac{T}{\chi(T)}}+\left[10+8.99 \ln \left(\frac{T}{\chi(T)}\right)\right] \\
& \times\left(\frac{T}{\chi(T)}\right)-17.5\left(\frac{T}{\chi(T)}\right)^{3 / 2},
\end{aligned}
$$

where $\chi(T)$ is the electrical conductivity in $\Omega^{-1} \mathrm{~m}^{-1}$, which is defined as the inverse of resistivity $\widetilde{\rho}(T)$. As in Fig. 3 it has been shown with experimental results 6] that a very good approximation can be obtained considering only the first term in Eq. (6):

$$
\varepsilon(T)=7.66 \sqrt{\frac{T}{\chi(T)}} .
$$

The electrical resistivity of most materials changes with temperature. Usually a linear approximation is used:

$$
\widetilde{\rho}(T)=\widetilde{\rho}_{0}\left[1+\alpha_{t}\left(T-T_{0}\right)\right] \simeq \frac{\widetilde{\rho}_{0}}{T_{0}} T
$$


67th International Astronautical Congress (IAC), Guadalajara, Mexico, 26-30 September 2016.

Copyright (C) 2016 by the International Astronautical Federation (IAF). All rights reserved.
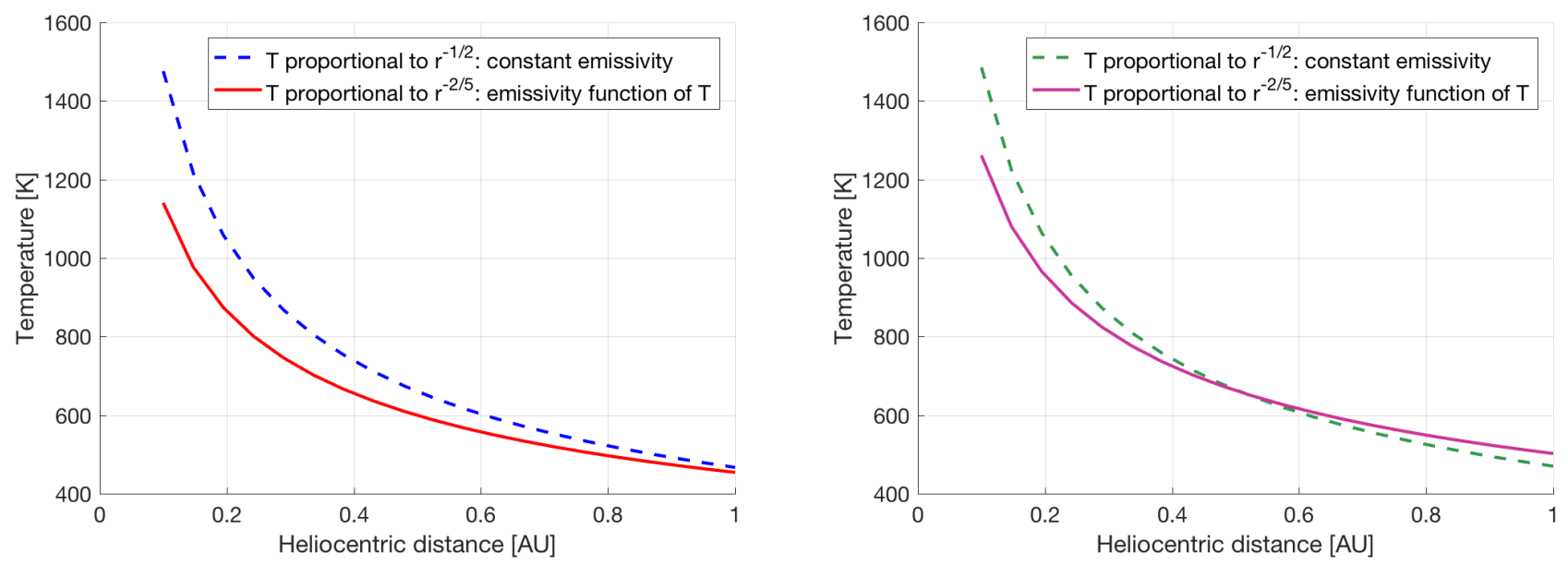

Figure 4: Dependence of material's temperature on the heliocentric distance for aluminum (left) and titanium (right), both for constant and variable emissivity.

where $\alpha_{t} \simeq 1 / 273 K^{-1}$ is called the temperature coefficient of resistivity, $T_{0}$ is a fixed reference temperature (commonly room temperature) and $\widetilde{\rho}_{0}$ is the resistivity at temperature $T_{0}$. Values for electrical conductivity, resistivity and temperature coefficient of various materials can be found in literature. As $\chi(T)$ is almost inversely proportional to the temperature, this means that $\varepsilon(T) \propto T$ :

$$
\varepsilon(T)=7.66 \sqrt{T \cdot \widetilde{\rho}(T)}=7.66 T \sqrt{\frac{\widetilde{\rho}_{0}}{T_{0}}} .
$$

Hence, by introducing emissivity dependence on temperature in Eq. (4), the temperature of the spacecraft varies as $r^{-2 / 5}$.

\subsection{Results for aluminum and titanium}

In this study the constant optical coefficient were taken from [6]. Experimental data for aluminum are $\rho=$ 0.88 and $\varepsilon=0.03$, whereas $\rho=0.22$ and $\varepsilon=0.19$ for titanium. For aluminum the coefficient in (7) is $7.52 K^{-1 / 2} \Omega^{-1 / 2} m^{-1 / 2}$, instead for titanium the same given in Eq. (7) was used. Considering a reference temperature of $T_{0}=293 \mathrm{~K}$, the resistivity for aluminum and titanium are $\widetilde{\rho}_{0}=2.82 \cdot 10^{-8} \Omega \cdot m$ and $\widetilde{\rho}_{0}=4.2 \cdot 10^{-7} \Omega \cdot m$, respectively.

Dependence of temperature on the heliocentric distance for a case of constant emissivity and, conversely, when emissivity of the metal depends on temperature is shown in Fig. 4, and listed in Table1. It is clear that, considering the temperature dependence of emissivity (solid curve), the object's temperature increases more slowly than in the case of constant emissivity (dashed curve), as the body approaches the Sun [1].

Table 1: Temperature dependence of an object on the heliocentric distance for aluminum and titanium considering emissivity constant or function of temperature itself.

\begin{tabular}{|c|cc|cc|}
\cline { 3 - 5 } \multicolumn{1}{c|}{} & \multicolumn{2}{|c|}{$T[\mathrm{~K}] \mathrm{Al}$} & \multicolumn{2}{c|}{$T[\mathrm{~K}] \mathrm{Ti}$} \\
\cline { 1 - 3 } & $T \propto r^{-1 / 2}$ & $T \propto r^{-2 / 5}$ & $T \propto r^{-1 / 2}$ & $T \propto r^{-2 / 5}$ \\
\cline { 1 - 3 } & $\varepsilon=$ const & $\varepsilon(T)$ & $\varepsilon=$ const & $\varepsilon(T)$ \\
\hline 0.1 & 1476.1 & 1140.6 & 1485.7 & 1261.3 \\
0.2 & 1043.8 & 864.4 & 1050.6 & 955.9 \\
0.3 & 852.2 & 735.0 & 857.8 & 812.8 \\
0.4 & 738.1 & 655.1 & 742.9 & 724.4 \\
0.5 & 660.1 & 599.2 & 664.4 & 662.6 \\
0.6 & 602.6 & 557.0 & 606.6 & 616.0 \\
0.7 & 557.9 & 523.7 & 561.6 & 579.1 \\
0.8 & 521.9 & 496.5 & 525.3 & 549.0 \\
0.9 & 492.0 & 473.6 & 495.2 & 523.8 \\
1 & 466.8 & 454.1 & 469.8 & 502.1 \\
\hline
\end{tabular}

\section{Future development}

Even though aluminum and titanium are the conventional materials for flight structures, graphitefiber/polymer-matrix composite materials are the most 
67th International Astronautical Congress (IAC), Guadalajara, Mexico, 26-30 September 2016.

Copyright (C) 2016 by the International Astronautical Federation (IAF). All rights reserved.

probable candidates for the future of aerospace industry. Carbon-fiber composite materials are easier to shape and lighter than metals, with much higher strength to density ratio [8]. Significant weight savings ( 25 to 50 percent) could be achieved through the use of polymer-matrix composites. However, a great engineering effort is required to establish confidence in their use. Our analysis could be easily extended to these materials, once their optical properties are known. Nevertheless, due to the unicity of each composite material, experimental values may be required.

\section{Conclusions}

Within the standard approach the reflectivity and emissivity of materials used in aerospace industry are conserved as constant values. As a result the temperature of the material increases when the spacecraft approaches to the sun as $T \sim r^{-1 / 2}$, where $r$ is the heliocentric distance. In our approach we consider the temperature dependence of the electro-optical parameters of materials that have implicit dependence on the temperature through the temperature dependence of the electrical conductivity of metals. It is shown that the temperature dependence of the emissivity and conductivity leads to the dependence of temperature for the materials used in aerospace industry approximately as $T \sim r^{-2 / 5}$ with the heliocentric distance. The proposed study compares the temperature dependence on heliocentric distance for metals (aluminum and titanium in particular) when their optical properties are considered constant or function of temperature. For a near-Sun mission it is of crucial importance to understand as much as possible the behavior of the spacecraft materials, as the greatest constraint will be the maximum temperature at which it can be fully functional and operative.

In our study the temperature reached at $0.1 \mathrm{AU}$ with variable emissivity is lower than when one assumes that the emissivity is constant, both for $\mathrm{Al}$ and $\mathrm{Ti}$. In other words, the spacecraft could approach closer to the Sun than it was assumed before based on the consideration that emissivity of materials used in aerospace indus- try does not depend on temperature. However, note that the titanium graph shows an intersection between the two curves. This indicates that there is no common behavior and the study must be carried out with a complete set of the chosen material's electro-optical parameters.

\section{Acknowledgements}

This research was supported by PSC CUNY Grant: award \# 68298-0046.

\section{References}

[1] R. Ya. Kezerashvili. Space exploration with a solar sail coated by materials that undergo thermal desorption. Acta Astronaut., 117:231-237, 2015.

[2] M. Peters and C. Leyens. Aerospace and space materials. Materials Science and Engineering.

[3] Y. A. Çengel and A. J. Ghajar. Heat and Mass Transfer - Fundamentals and Applications. McGraw-Hill Education, 5th edition, 2015.

[4] A. E. Roy and D. Clarke. Astronomy: Principles and practice. Taylor and Francis, Fourth Edition, 2003.

[5] R. Ya. Kezerashvili. Solar sail: Materials and space environmental effects.in the book: M. Macdonald (Ed.), Advances in Solar Sailing, Springer Praxis Books, Berlin, Heidelberg, pages 573?592, 2014.

[6] M. F. Modest. Radiative Heat Transfer. McGrawHill, 1993.

[7] W. J. Parker and G. L. Abbott. Theoretical and ex- perimental studies of the total emittance of metals. Symposium on Thermal Radiation of Solids, NASA SP-55, pages 11?28, 1965.

[8] National Research Council L. J. Adams. Technology for small spacecraft. The Aeronautics and Space Engineering Board, 1994. 